\title{
Synthesis of malonyldi(1,2,4-triazole), the study of the kinetic and thermodynamic features of its hydrolysis and prediction of biological activity
}

\author{
C Pyotr P. Purygin, ${ }^{1}{ }^{+}$Maria V. Evstegneeva, ${ }^{1}$ Roman V. Shafigulin, ${ }^{2}$ and Yury P. Zarubin ${ }^{1}$ \\ ${ }^{1}$ Department of Inorganic Chemistry. ${ }^{2}$ Department of Physical Chemistry and Chromatography. \\ Natural Science Institute. Samara National Research University. Akademika Pavlova St., 1. \\ Samara, 443011. Samara Region. Russia. Phone: ${ }^{1)}+7$ (846) 334-54-59, ${ }^{2)}$ (846) 334-54-47. \\ E-mail:1) puryginpp2002@mail.ru,evstegneeva.starkova@yandex.ru,yupzarubin@mail.ru; \\ 2)shafiro@mail.ru
}

* Supervising author; ${ }^{+}$Corresponding author Keywords: malonyldi(1,2,4-triazole), synthesis, hydrolysis kinetics, hydrolytic stability, hydrolysis rate constant, half-life, thermodynamic characteristics, calculations, quantum-chemical, molecularmechanical, biological activity, prediction.

\begin{abstract}
This article describes a two-step method for the synthesis of malondyl (1,2,4-triazole) (1,3-di (1H-1,2,4triazol-1-yl)propane-1,3-dione) of 1,2,4-triazole through 1-trimethylsilyl-1,2,4-triazole, followed by reaction with malonyl chloride; the yield of the final product is $85 \%$. The kinetics of hydrolysis of malonyldi $(1,2,4-$ triazole) in the acetonitrile - water system $(9: 1)$ at $25{ }^{\circ} \mathrm{C}$ and $35^{\circ} \mathrm{C}$ was studied, and the activation energy of the hydrolysis of malonyldi(1,2,4-triazole). To study the thermodynamic features of the reactions of obtaining this compound in the Spartan'14 1.1.4 program, a number of thermodynamic characteristics were calculated, which determine the spontaneous and exothermic nature of the process.

For the molecule malonyldi(1,2,4-triazole), the most and least stable conformers were found in the program Molecular Operating Environment 2009.10, for which the surfaces of nucleophilic susceptibility were calculated in the program SCIGRESS Modeling 3.1.4. It was shown that the most stable conformer of the malonyldi(1,2,4-triazole) molecule should have the highest reactivity in reactions with different nucleophiles and the lowest value of the heat of formation. In this case, the nucleophilic susceptibility of carbonyl carbon atoms is different, despite the symmetry of the structure of the molecules malonyldi(1,2,4triazole), which suggests a stepwise interaction of malonyldi(1,2,4-triazole) with nucleophiles.

The structure of malonyldi(1,2,4-triazole) was confirmed by IR, ${ }^{1} \mathrm{H}$ NMR spectroscopy, individuality by thin-layer chromatography. The proposed mechanism for the hydrolysis of malonyldi(1,2,4-triazole) is described. The program PASS Professional 2007 predicted the most likely types of biological activity of the studied compounds. The most significant types of biological activity are anti-diabetic, anti-ulcer, antiischemic, anti-oxidant. The obtained data allow us to choose the optimal conditions for the synthesis of malonyldi(1,2,4-triazole) and to conclude that it is highly hydrolytically stable in an aqueous acetonitrile medium.
\end{abstract}

\section{References}

[1] G.I. Kappan, S.S. Kukalenko. Triazoles and their pesticidal activity. Moscow: NIITEKHIM. 1983. 70p. (russian)

[2] http://www.rlsnet.ru

[3] I.A. Abdugafurov, N. Madikhanov, A.G. Makhsumov, H.M. Shahidoyatov, A.A. Muzaffarov, A.U. Zokirov, Sh.S. Masharipova. Anti-inflammatory activity of 1,2,3-triazole derivatives / "Chemistry and biological activity of heterocycles and alkaloids": First International Conference. October 9-12, 2001, Moscow: Abstr. Pap. Moscow. 2001. P. 8-9. (russian)

[4] V. Prachayasittikul, R. Pingaew, N. Anuwongcharoen, A. Worachartcheewan. J. Springer. 2015. No.4. P.1-22.

[5] V.S. Talismanov, S.V. Popkov. Agrochemistry. 2007. No.5. P.53-57. (russian)

[6] A.G. Mazharova. Nitrogen heterocycles in medicine and agriculture: a training manual. Novocherkassk: South-Ros. State Polytechnic University (NPI). 2013. 79p. (russian) 
[7] G. Singh, K. Chowdhary, P. Satija, A. Singh, B. Singh, K. Singh, C. Espinosa, M.A. Esteban, R. Sehgal, V. Verma. Biological Chemistry and Chemical Biology. 2018. No.3. P.1609-1614.

[8] A-R. Farghaly, H. El-Kashef. General Papers. 2006. No.6. P.76-90.

[9] https://www.chemicalbook.com/ChemicalProductProperty_EN_CB515 2018. htm

[10] P.P. Purygin, V.Yu. Alekseev, I.N. Alekseev, E.A. Agapova, and Yu.P. Zarubin. Preparation of $N, N^{\prime}-$ oxalyldiimidazole and investigation of its interaction with alkylamines and succinic acid. Butlerov Communications. 2018. Vol.54. No.6. P.111-115. DOI: 10.37952/ROI-jbc-01/18-54-6-111 